\title{
Glosim: Global System Image for Cluster Computing
}

\author{
Hai Jin, Li Guo, Zongfen Han \\ Internet and Cluster Computing Center \\ Huazhong University of Science and Technology, Wuhan, 430074, China
}

\begin{abstract}
This paper presents a novel architecture of a single system image built on cluster system, named Glosim. This system orientates to cluster system middleware, which provides global system image support. It not only supports global IPC objects including message queue, semaphore and shared memory, but also presents a new concept of global working process and provides it SSI support smoothly and transparently. Combined with Linux Virtual Server, single IO space, it completely constructs a high performance SSI cluster network server.
\end{abstract}

Keywords: Cluster, Single System Image, Global Working Process, IPC

\section{Introduction}

A single system image (SSI) [1][2][7][8][9] is the property of a system that hides the heterogeneous and distributed nature of the available resources and presents them to users and applications as a single unified computing resource. SSI can be enabled in numerous ways, ranging from those provided by extended hardware to various software mechanisms. SSI means that users have a global view of the resources available to them irrespective of the node to which they are physically associated. Furthermore, SSI can ensure that a system continues to operate after some failure (high availability) as well as ensuring that the system is evenly loaded and providing communal multiprocessing (resource management and scheduling).

SSI design goals for cluster-based systems are mainly focused on complete transparency of resource management, scalable performance, and system availability in supporting user applications. A SSI can be defined as the illusion, created by hardware or software, which presents a collection of resources as one more powerful unified resource.

In this paper, we present a novel architecture of a single system image built on cluster system. Our purpose is to provide system level solution needed for a single system image on 
high performance cluster with high scalability, high efficiency and high usability. Currently cluster of net server style still looks like a collection of PCs on a network. Global System Image (Glosim) aims to solve these issues by providing a mechanism to support global IPC (Inter-Process Communication) objects including message queue, semaphore and shared memory and by making all the working process visible in the global process table on each node of clusters. The hope is that it will completely meet the need of people to be able to visit a cluster as a single server. That is to smoothly migrate Unix programs from traditional single machine environment to cluster environment almost without modification.

The paper is organized as follows: Section 2 provides the background and related works on single system image in cluster. Section 3 describes a generic and scalable global system image project overview. Section 4 describes Glosim software architecture and introduces the global IPC and global working process. In Section 5, we list the implementation of Glosim including software infrastructure, error detection, and security. Section 6 presents analysis of performance effects on the developed prototype of the Glosim. Section 7 describes the Glosim enabled applications supported by Glosim Cluster. Finally, a conclusion is made in Section 8 with more future work.

\section{Related Works}

Research of OS kernel-supporting SSI in cluster system has been pursued in a number of other projects. Below we briefly examine the work performed in this area in SCO UnixWare [3], Mosix [4][16], Beowulf Project [17][18], Sun Solaris-MC [5][19] and GLUnix [6].

\subsection{SCO UnixWare}

UnixWare NonStop Clusters is SCO's high-availability software. It significantly broadens hardware support, making it easier and less expensive to deploy the most advanced clustering software for Intel systems. It is an extension to the UnixWare operating system in which all applications run better and more reliably inside a SSI environment that removes the management burden. The UnixWare kernel has been modified via a series of modular extensions and hooks to provide single cluster-wide file system view, transparent cluster-wide device access, transparent swap-space sharing, transparent cluster-wide IPC, high performance internode communications, transparent cluster-wide process migration, node down cleanup and resource fail-over, transparent cluster-wide parallel TCP/IP networking, application availability, cluster-wide membership and cluster time sync, cluster system administration, and load leveling.

UnixWare NonStop Clusters also supports active process migration, which allows any application process to be moved to another node between instruction steps. This allows 
continuation without disruption to the application. Active process migration allows dynamic removal and addition of nodes within the cluster.

\subsection{Mosix}

Mosix is a clustering project at the Hebrew University of Jerusalem, which is not based purely on Linux. Mosix has been developed a number of times for UNIX, BSD and, more recently, Linux. The system provides the transparent migration of processes between nodes in the cluster to achieve a balanced load across the cluster. The system is implemented as a set of adaptive resources sharing algorithms, which can be loaded into the Linux kernel using kernel modules. The algorithms attempt to improve the overall performance of the cluster by dynamically distributing and redistributing the workload and resources among the nodes of a cluster of any size.

\subsection{Beowulf Project}

The Beowulf Project is initiatively undertaken by NASA to develop an effective cluster computing solution to NASA's computational requirements in Earth and space sciences. The first Beowulf cluster was built in 1994 by Thomas Sterling and Donald Becker and consisted of 16 DX4 processors connected with channel-bonded Ethernet1. Since then Beowulf clusters have become increasingly popular, especially for large-scale academic and commercial scientific applications.

Today Beowulf is a term that refers to a general class of clusters built on similar principles to the original Beowulf cluster. These principles include: The cluster is built for speed, not reliability, built from off the shelf, commodity hardware. Each node runs a free-software operating system like Linux or Free-BSD. The cluster may run a modified kernel allowing channel bonding, a global PID space [18] or DIPC [12][13][14][20] to be used. A global PID space lets you see all the processes running on the cluster with ps. And DIPC allows you to use shared memory, semaphores and wait-queues across the cluster. The clusters generally run software written using parallel programming libraries like MPI or PVM.

\subsection{Sun Solaris MC}

Solaris MC is a prototype distributed operating system developed at Sun Microsystems Laboratories. The system extends the Solaris UNIX operating system using object-oriented techniques. To achieve these design goals each kernel subsystem was modified to be aware of the same subsystems on other nodes and to provide its services in a locally transparent manner. For example, a global file system called the proxy file system, or PXFS, a distributed pseudo /proc file-system and distributed process management were all implemented.

These global subsystems were implemented as a set of components built on top of the 
base kernel using the CORBA object model. By using CORBA the subsystem interfaces could easily be provided to the entire distributed system. Also, by abstracting components of the kernel using CORBA's interface definition language (IDL) it was possible to use an IDL compiler to create the glue needed to perform arbitrary service requests on these components.

\subsection{GLUnix}

Another way for the operating system to support a SSI is to build a layer on top of the existing operating system and to perform global resource allocations. This is the approach followed by GLUnix from Berkeley. This strategy makes the system easily portable and reduces development time. GLUnix is an OS layer designed to provide support for transparent remote execution, interactive parallel and sequential jobs, load balancing, and backward compatibility for existing application binaries. GLUnix is a multi-user system implementation at the user level so that it can be easily ported to a number of different platforms. It is built as a protected user-level library using the native system services as a building block.

GLUnix aims to provide cluster-wide name space and uses network PIDs (NPIDs) and virtual node numbers (VNNs). NPIDs are globally unique process identifiers for both sequential and parallel programs throughout the system. VNNs are used to facilitate communications among processes of a parallel program. A suite of user tools for interacting and manipulating NPIDs and VNNs is supported. The main features provided by GLUnix include co-scheduling of parallel programs; idle resource detection, process migration, and load balancing; fast user level communication; remote paging; and availability support.

Aiming at the above backgrounds, based on PC Cluster, this paper analyzes and studies the designing contents, key technologies and implementing methods of single system image layer in cluster operating system and then puts forward the concept of global system image for cluster system. What's more, according to the application background of cluster system in network services, it also introduces a new concept of global working process, designs and implements Global System Image (Glosim), trying to realize single system image in certain degree through cluster system middleware.

\section{System Overview}

Glosim is a single system image implementation prototype based on Linux cluster. It is mainly used to construct high performance cluster, such as distributed web server, distributed BBS server, distributed MUD server and so on. The designing aim of Glosim is to conveniently migrated UNIX programs from former single machine environment to distributed cluster environment without too much modification. For example, former FireBird BBS can be easily migrated to Glosim distributed cluster environment just by disposing 
global variables.

Figure 1 is the complete infrastructure of Glosim. Combined with Linux Virtual Server [10], SIOS [11][12], it completely constructs a high performance SSI cluster network server.

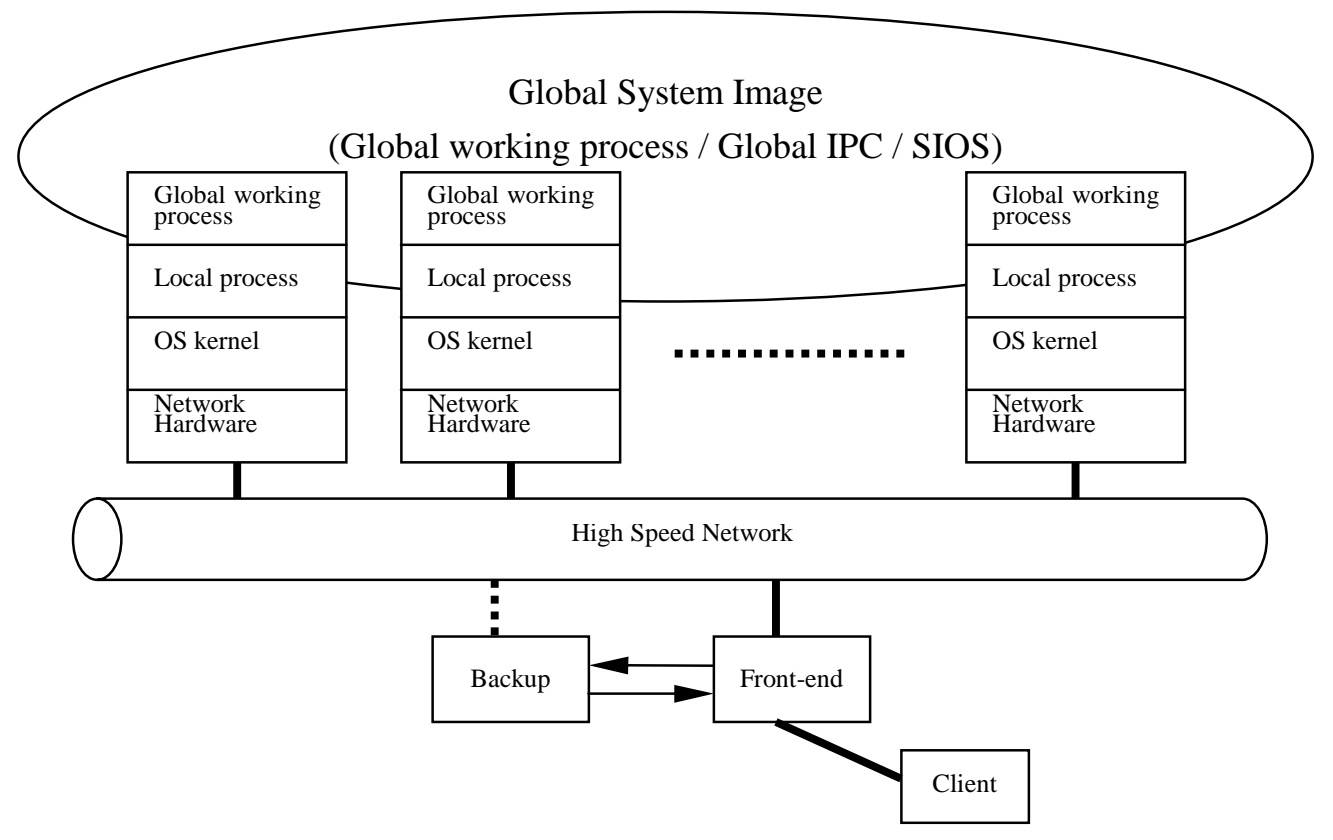

Figure 1 Glosim Software Architecture

Cluster network server based on Glosim is constructed by some PCs connected by switch to provide high load network services. It consists of three major parts with three logical layers.

The first layer includes Front-end and Backup, which run Linux Virtual Server to schedule and backup each other to improve system reliability. They are the message entrance to the whole server in charge of receiving client requests and distributing these requests to the processing nodes of the cluster according to certain strategy (such as WRR [10] algorithm). Because Front-end and Backup are only in charge of redirections requested by clients and do not involves data processing, they are quite slightly loaded. Besides, in Front-end and Backup, hot standby daemon is running for fault tolerance. In case of failure in Front-end, Backup can take charge of its functions by robbing IP and recover Front-end kernel data to achieve high reliability of the whole system network connection.

The second layer are local OS kernel and local processes of the processing nodes inside cluster, which are in charge of system booting, providing basic system call and application software support. Logically, they are outside the boundary of SSI.

The third layer is Glosim layer, as middleware of cluster system, which includes global IPC, global working process, SIOS. Logically, this layer is inside the boundary of SSI and 
provides SSI service to all the working processes of cluster system.

\section{Glosim Software Architecture}

We have analyzed DIPC prototype, the typical SSI software in Linux environment and then do some emendation and expansion to DIPC and Linux kernel. In this way, we primarily implement Glosim software prototype supporting cluster system.

An overview of Glosim software architecture is shown in Figure 1 above. It includes the following two major components: global IPC and global working process.

\subsection{Global IPC}

Linux supports three types of inter-process communication mechanism, which are respectively message queue, semaphore and shared memory. These three communication mechanisms first appear in Unix TM System V using the same authorization method. Processes can only access these materials by transferring a unique reference designator to the kernels through system invokes.

Global inter-process communication IPC mechanism is just the globalization of IPC objects, which is mainly implemented based on DIPC. Through DIPC, it provides the whole cluster with global IPC, including consistency of shared memory, message queue and semaphore.

What should be specially pointed out here is that Distributed Shared Memory [1][2] in global IPC uses a multiple-read / single-write protocol. Global IPC replicates the contents of the shared memory in each node with reader processes, so they can work in parallel, but there can be only one node with processes that write to a shared memory. The strict consistency model is used here, meaning that a read will return the most recently written value. It also means that there is no need for the programmer to do any special synchronization activity when accessing a distributed shared memory segment. The most important disadvantage with this scheme is a possible loss of performance in comparison to other DSM consistency models. Global IPC can be configured to provide a segment-based or a page-based DSM. In the first case, DIPC transfers the whole contents of the shared memory from node to node, with no regard to whether all that data are to be used or not. This could reduce the data transfer administration time. In the page-based mode, $4 \mathrm{~KB}$ pages are transferred as needed. This makes multiple parallel writes to different pages possible. In global IPC, each node is allowed to access the shared memory for at least a configurable time quantum. This lessens the chances of the shared memory being transferred frequently over the network, which could result in very bad performance. 
The establishment of global inter-process communication inside cluster system provides the basis for data transfer between processes in each node of the cluster. The programmer can use standard inter-process communication functions and interface of System $\mathrm{V}$ to conveniently write application programs suitable to cluster system. What's more, former programs can be migrated to Glosim without too much payout. Actually, Glosim enabled applications only require the programmer to do some special transactions to global variables. For example, global variables can be stored as structures in shared memory, and accessed normally.

\subsection{Global Working Process}

This part is divided into the following four sections: (a) Concept of Global Working Process, (b) Global Working Process Space, (c) Global Signal Mechanism, (d) Global Proc File System.

\section{(a) Concept of Global Working Process}

Considering a practically running cluster system, it is necessarily a server providing single or multiple services and we just call these serving processes working process. What we should do is to include working processes to the boundary of SSI and provide them SSI service from system level. In this way, working processes in different nodes can communicate transparently and become global working processes.

However, not all processes in each node are necessarily to be actualized with SSI in the whole cluster system. That is to say, only some of the processes reside within the boundary of SSI. By introducing the concept of working process, we may solve the inconsistency of OS system processes between each node. OS system process of each node is relatively independent, outside SSI boundary while global working process is provided by the system with single system image service, inside SSI boundary. From the view of long range, it also affords a good foundation for the cluster system to extend to OS heterogeneous environment.

As for practical programming implementation, it is also quite easy to express working process. For example, we can tag working process with a special uid for the convenience of kernels recognition and independent transaction. This enactment is closely related to the practical system. For instance, httpd process in Apache is often running as an apache user and bbsd process in BBS is commonly executed as a bbs user.

(b) Global Working Process Space

Single process space has the following basic features:

- Each process holds one unique pid inside the cluster.

- A process in any node can communicate with other processes in any remote node by 
signals or IPC objects.

- The cluster supports global process management and allows the management of all working processes just like in local host.

In order to uniquely tag the working process in the whole cluster, we must introduce the concept of global working process space. There are mainly two solutions as follows:

- Algorithm 1: modulus the process number to tag the node number in which the process runs. For example, in a cluster system with 16 nodes, the way of calculating the node number of process 10001 is $10001 \% 16=1$. So process 10001 is running in node 1 .

- Algorithm 2: distribute different working process pid space to each node. For instance, the working process space of node 1 is between 10000 and 20000, the working process space of node 2 is between 20000 and 30000, and so on.

No matter which algorithm we adopt, the purpose is to make working process pid unique within the whole cluster. As for each node, local process pid is decided by last_pid of OS kernel itself. Each node is independent on each other but every local pid is smaller than the lower limit of global process space fixed in advance. In short, local process pid is below, independent on each other while global working process pid is above, unique in the whole cluster.

(c) Global Signal Mechanism

Signal is the oldest inter-process communication mechanism in Unix system, used to transfer asynchronous signals. In global signal mechanism, all working processes can send each other signals using the functions like signal (), kill () and so on just like in the same computer. That is to say, processes can transparently conduct mutual communication within the cluster by using functions like signal (), kill () and so on.

For example, Glosim deals with system invoke kill (pid, signal) as follows:

Step 1: Check uid of the current process to see whether it is a global working process;

Step 2: If TRUE continue, else goto Step 9;

Step 3: Calculate the corresponding node number of the object pid;

Step 4: IF local host, goto Step 9; IF other nodes, continue;

Step 5: Transfer to Back-end daemon [see Figure 3] and the system call return;

Step 6: Back-end daemon produces Employer process;

Step 7: Transfer to the target node; 
Step 8: Front-end daemon [see Figure 3] of the target node receives, transacts and handles;

Step 9: Transfer to OS kernel to naturally deal with continually and the system call return;

Its transaction flow chart is as follows:

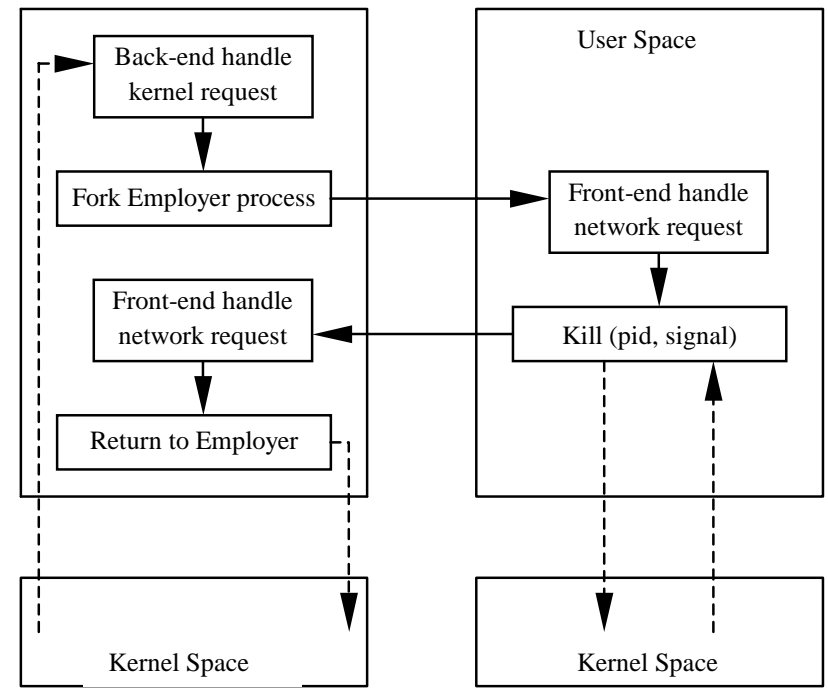

Figure 2 Processing Flow of Glosim

(d) Global Proc File System

Global file system is to map status messages of all processes in all nodes to different nodes for the convenience of programmer and system administrator's controlling. Global proc file system ignores the processes with the process number smaller than max_local_pid, which are regarded as local processes. This is because Glosim system aims to actualize the single system image of working processes and one basic characteristic of working processes is the process number bigger than max_local_pid. So we can only consider working processes.

With the actualizing of global proc file system, we can use ps -ax command to examine local processes running in current node and all global working processes in the whole Glosim system. What's more, we can employ kill command to send signals to any process and even adopt kill -9 command to end any process we want with no regard to which node it actually runs. In this way, complete working process single image is implemented in cluster system.

\section{Implementation of Glosim}

\subsection{Software Infrastructure}

Glosim has two parts: The main part, a program called glosimd, running in the user space 
with root privileges, and another part, hidden inside the Linux kernel, which allows the first part to access and manipulate kernel data. This design consults DIPC prototype source code and is the result of the desire to keep the needed changes in the operating system kernel to a minimum.

glosimd creates several processes to do this work. These processes manage all the necessary decision makings and network operations. They use predetermined data packets to communicate with glosimd processes on other nodes. In these packets, all the necessary information for a requested action, including the parameters to a system call, as obtained from inside the kernel, are included.

The whole system implementation infrastructure is as follows:

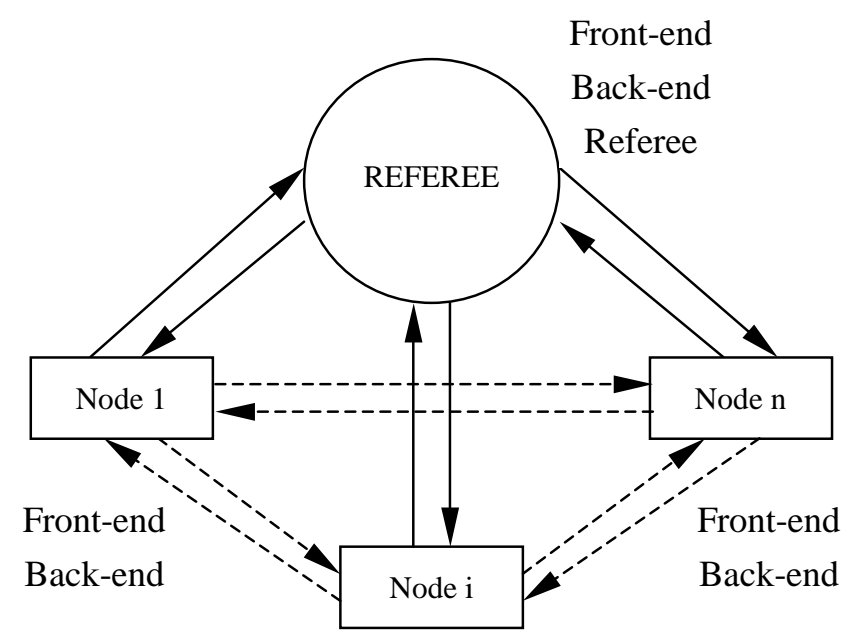

Front-end

Back-end

Figure 3 System Implementation Infrastructure

glosimd is divided into three major modules: Front-end, Back-end and Referee. Among those, Front_end runs in every node, in charge of transacting data requests from network and forking worker process. Back-end also runs in every node, in charge of transacting data request from kernel and forking employer process. Referee, as the central arbitrating node, only runs in the central node, maintaining a chain table of all IPC structures and pid. Employer, forked by Back-end, executes remote system invokes. Worker, forked by Front-end, transacts remote requests from other nodes.

\subsection{Error Detection}

Glosim adopts a fail-stop distributed environment. So it uses time-outs to find out any problem. Here the at-most-once semantics is used, meaning that Glosim tries everything only once. In case of an error, it just informs it of the relevant processes, either by a system call return value, or, for shared memory read/writes, via a signal. Glosim itself does not do 
anything to overcome the problem. It is the user processes that should decide how to handle it. This is the normal behavior in many other cases in Unix.

\subsection{Security}

It is very important to provide some means to make sure that only people who are allowed to do it can access the data. Glosim uses login names to identify users. Remote operations are performed after assuming the identity of the person that executed the system call originally. To achieve this, the same login name on all the computers in a Glosim Cluster should denote the same operation. Besides, in order to prevent intrusion to Glosim Cluster, addresses of the nodes that are allowed to take part in a cluster should be put in a local configuration file for Glosim to consult.

\section{Analysis of Performance Effects}

This section presents some basic analytical results on the performance of the Glosim Cluster. The benchmark program sets up a shared memory, a message queue, and a semaphore set. The tested system calls are: semctl(), msgctl(), shmctl() with the IPC_SET command, semop() and kill() operations. Besides, msgsnd() and msgrcv() with 128, 256, 512, 1024, 2048 and 4096 bytes messages are also tested.

Figure 4 is an experiment conclusion to measure the speed of executing some of the Glosim system calls in your system. It shows the executing times of semctl(), msgctl(), shmctl()with the IPC_SET command, semop(), and kill() in practical system benchmark with the unit ms.

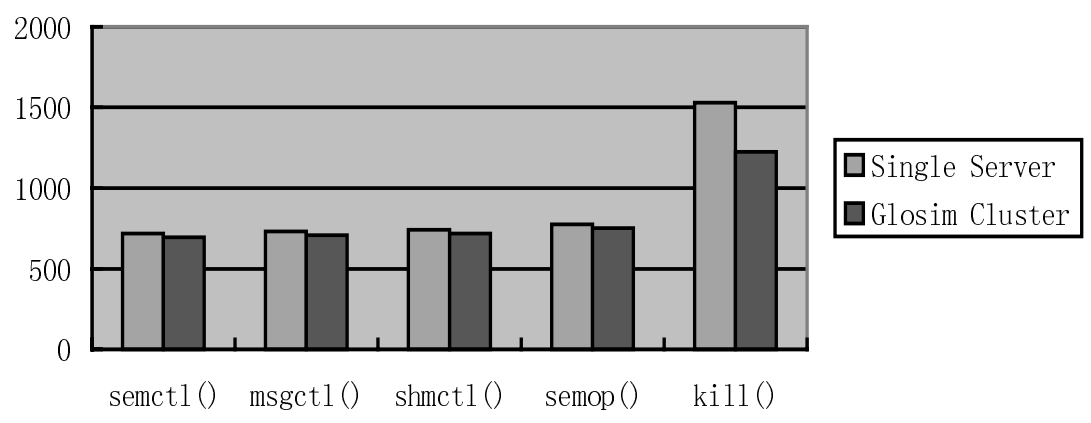

Figure 4

Figure 5 and 6 are some experiment conclusions to measure the speed of send and receive message in the system. Figure 5 shows the system message transfer bandwidth with the message size of 128, 256 up to 4096 bytes in single server. Figure 6 shows the system message transfer bandwidth with the message size of 128, 256 up to 4096 bytes in Glosim server with 8 nodes. 


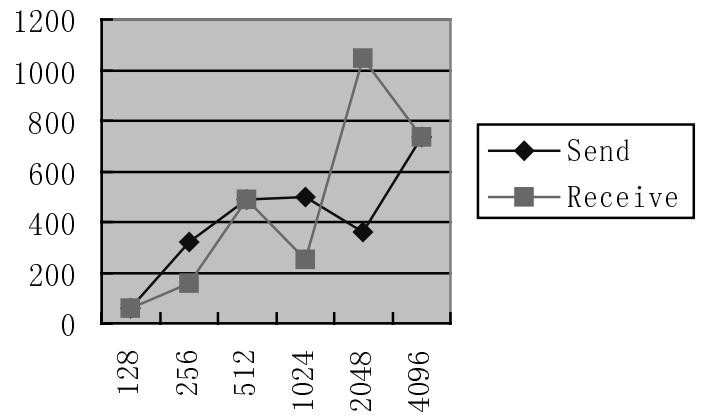

Figure 5 Single Server

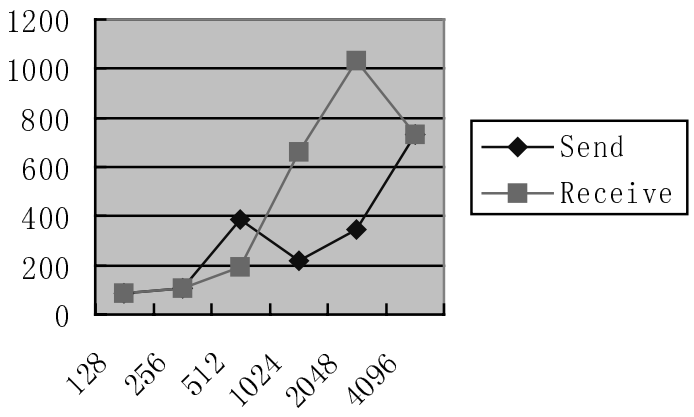

Figure 6 Glosim Server with 8 Nodes

As the data shown in figure 4, Glosim has a normal and acceptable response speed to atom operation of IPC objects and global signal processing mechanism. According to the data shown in the figure 5 and 6 , in case of low data load (4KB below), msg send/receive bandwidth in Glosim server is a little smaller than that in single server. The results appear to be quite normal and acceptable because Glosim server involves network transfer and the overload of data copy of kernel and user space. Thus, by the above three figures, Glosim also has excellent performance support for practical system.

\section{Glosim Enabled Applications}

In fact, programs based on Glosim can exchange IPC objects just by numerical keys and they can tag each global working process using global process pid. This indicates that there is no need for them to make sure where the corresponding IPC structure lies physically and in which node the practical global working process runs. Glosim system ensures that working processes can find out the needed resources and other working processes only by fixed key values and global pid. These resources and working processes lie in different nodes when running. However, through the logical addressing and global pid addressing of resources, programs can be independent on any physical network characteristics and thus transparently actualize single system image for upper application programs.

Glosim just aims to smoothly migrate Unix programs from traditional single machine environment to cluster environment almost without modification. So, as a traditional Unix program, BBS is quite a typical example which involves different aspects of Unix including network socket, IPC object, signal operation, file I/O operation and so on. What's more, it is very popular and much requires serving efficiency after login. If supported by Glosim, BBS can be smoothly migrated to cluster environment with considerable performance improvement compared with that in single environment, it fully proves that Glosim is of high usability, performance and stability. 
Scalable BBS cluster server is a practical application based on Glosim. In order to efficiently extend the traditional BBS with single server to the BBS with cluster server and provide services of high performance, file consistency, balanced load and transparency, we make the design requirement as follows:

(1) BBS original program based on IPC mechanism in former single environment must be extended to the distributed one.

(2) Materials in shared memory and file system of each node in cluster must keep consistent.

(3) Servers can be increased and deleted dynamically or statically.

(4) Numbers of the registration and the login must increase in proportion to the numbers of servers.

(5) Support Linux Virtual Server to complete load balancing between servers.

(6) Transparently support upper applications from system layer almost without modifying user program codes.

Figure 7 shows the max login numbers of BBS in single server with different memory settings. And figure 8 shows the max login numbers of BBS in Glosim cluster with different memory settings and different nodes. $\mathrm{X}$-axis indicates the number of nodes, while Y-axis indicates the numbers of people.

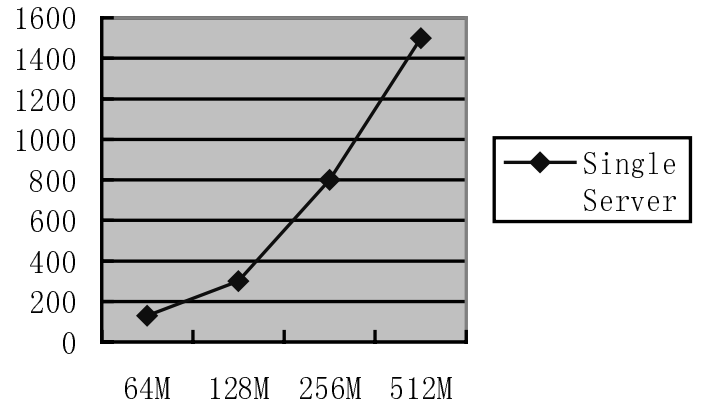

Figure 7 (Single Server)

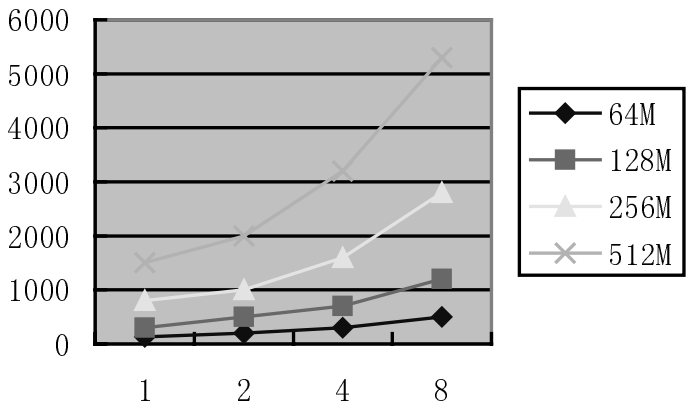

Figure 8 (Glosim Server)

\section{Conclusions and Future Works}

This paper has attempted to analyze more than is usually done the notion of a "single system image". DIPC system that we consulted only conducts distributing work based on IPC mechanism, but doesn't realize complete single system image. In this paper, we present a novel architecture of a single system image built on cluster system, named Glosim. This 
system orientates to cluster system middleware, which provides global system image support. It not only supports global IPC objects including message queue, semaphore and shared memory, but also presents a new concept of global working process and provides it SSI support smoothly and transparently. Combined with Linux Virtual Server, SIOS, it completely constructs a high performance SSI cluster network server.

In this case, a great number of applications in single Linux environment such as BBS, Apache, MUD server can be migrated into cluster environment almost unmodified. In this way, single system image service is provided to upper application programs transparently.

With all its advantages mentioned, however, Glosim is utilized currently with the disadvantages of relatively high system overhead, fault tolerance to be improved and only indirect support to application program global variables. To achieve better performance, what we tend to make efforts for may be as follows: First, add the function of fault tolerance. The current idea is to create backup of Referee node and actively copy the IPC objects established in each node. In this case, the cooperation and consistency of the whole fault tolerance system can be achieved. Second, use KKT (Kernel to Kernel Transfer) to improve the Glosim performance. Ideally, a zero-copy protocol between two kernels should be used, whereby the message is moved directly from the send buffer in the source node kernel to the receive buffer in the destination node kernel, without being buffered in another memory region. Finally, try to make the system stable as quickly as possible and benchmark its performance and discuss and analyze it in theory.

\section{References}

[1] K. Hwang and Z. Xu, Scalable Parallel Computing: Technology, Architecture, and Programming, McGraw-Hill, New York, 1998.

[2] R. Buyya (ed), High Performance Cluster Computing: Architectures and Systems, Vol. 1., Prentice Hall, 1999.

[3] B. Walker, and D. Steel, "Implementing a full single system image UnixWare cluster: Middleware vs. underware", Proceedings of the International Conference on Parallel and Distributed Processing Techniques and Applications, (PDPTA'99)

[4] A. Barak, and O. La'adan, "The MOSIX multicomputer operating system for high performance cluster computing”, Future Generation Computer Systems, 1998

[5] Y. A Khalidi, J. M Bernabeu, V. Matena, K. Shirriff, and M. Thadani. "Solaris MC: A Multi-Computer OS”, Proceedings of 1996 USENIX Conference, January 1996.

[6] D. Ghormley, D. Petrou, S. Rodrigues, A.Vahdat, and T. Anderson, "GLUnix: A global 
layer Unix for a network of workstations”, Software Practice and Experience, 1998.

[7] R. S. C. Ho, K. Hwang; and H. Jin, "Towards a portable cluster computing environment supporting single system image", Proceedings. 1st IEEE Computer Society International Workshop on Cluster Computing, 1999, pp.158-166

[8] R. Buyya, "Single System Image: Need, Approaches, and Supporting HPC Systems", Proceedings of Fourth International Conference on Parallel and Distributed Processing Techniques and Applications (PDPTA'97), Las Vegas, Nevada, USA, 1997.

[9] R. Buyya, T. Cortes, and H. Jin, "Single System Image (SSI)", The International Journal of High Performance Computing Applications, Vol.15, No.2, Summer 2001, pp.124-135

[10] W. Zhang, "Linux virtual servers for scalable network services", Proceedings of Ottawa Linux Symposium 2000, Canada.

[11] K. Hwang, H. Jin, E. Chow, C. L. Wang, Z. Xu, "Designing SSI clusters with hierarchical checkpointing and single I/O space", IEEE Concurrency 7 (1): 60-69, 1999.

[12] K. Hwang; H. Jin, "Single I/O space for scalable cluster computing", Proceedings of 1st International Workshop on Cluster Computing, 1999, pp.158-166

[13] K. Karimi and M. Sharifi, "DIPC: A System Software Solution for Distributed Programming", Parallel and Distributed Processing Techniques and Applications Conference (PDPTA'97), Las Vegas, USA, 1997.

[14] K. Karimi and M. Sharifi, "DIPC: The Linux Way of Distributed Programming", Proceedings of the 4th International Linux Conference, Germany, 1997.

[15] K. Karimi, M. Schmitz and M. Sharifi, "DIPC: A Heterogeneous Distributed Programming System”, The Third International Annual Computer Society of Iran Computer Conference (CSICC'97), Tehran, Iran, 1997.

[16] MOSIX - Scalable Cluster Computing for Linux, http://www.cs.huji.ac.il/mosix/

[17] The Beowulf Project, http://www.beowulf.org/

[18] BPROC: Beowulf Distributed Process Space, http://www.beowulf.org/software/bproc.html

[19] Sun Solaris-MC, http://www.cs.umd.edu/ keleher/dsm.html

[20] DIPC, http://www.gpg.com/DIPC/ 